\title{
Développements récents dans la conception des turbines-pompes de hautes chutes
}

\author{
G. Vuillerod et A.P. Champier \\ GEC ALSTHOM NEYRPIC
}

L'accumulation d'énergie hydraulique par pompage continue à se développer pour les chutes moyennes et s'oriente depuis plusieurs années vers des hauteurs de chutes de plus en plus élevées à la recherche d'une réduction des investissements.

La modulation de la charge en turbine est affectée d'une valorisation importante. En effet les grands réseaux interconnectés, de par la nécessité de faire face aux besoins croissants d'énergie, présentent une évolution telle que la production thermique (d'origine classique ou nucléaire) peu ou pas réglable devient de plus en plus prépondérante devant la production hydraulique.

Les services d'exploitation attendent des usines de transfert d'énergie, qu'elles assurent la sécurité du réseau, c'està-dire :

- faire face aux fluctuations lentes et aléatoires de la consommation,

- éliminer les surcharges,

- enrayer les processus de dégradation de tension.

La conception et le dimensionnement des turbinespompes de haute chute nécessitent de la part du concepteur une attention toute particulière du fait des conditions sévères de fonctionnement de ces machines: démarrage et arrêts fréquents en mode turbine, démarrages en pompe, compensation synchrone, emballements...

Une conception optimale implique un compromis entre la recherche des performances, l'intégrité des structures, l'usinabilité, la facilité d'installation, la fiabilité à long terme et le coût de l'équipement.

Pendant le processus d'optimisation le constructeur doit par exemple déterminer le niveau des contraintes admissibles, la fréquence et l'amplitude des vibrations et des flexions. Les problèmes posés sont différents selon l'organe de la machine considéré. On peut distinguer en effet, deux modes de sollicitations, caractérisés essentiellement par la nature des forces mises en jeu:

- les charges statiques qui s'appliquent aux parties fixes du groupe,

- les charges dynamiques qui agissent surtout sur les organes de vannage, les aubages de la roue, la ligne d'arbre et les paliers.

La maîtrise de ces dernières pose les problèmes les plus difficiles à résoudre sur les turbines-pompes de très hautes chutes.

Les références de GEC ALSTHOM NEYRPIC (GANP) dans le domaine des turbines-pompes couvrent une très large gamme de hauteurs de chute, une grande diversité de puissances unitaires et de caractéristiques de fonctionnement.

En participant à plus de 25 grands projets de station de pompage, avec chaque fois la volonté de s'adapter aux attentes de ses clients par l'évolution constante de la conception hydraulique et mécanique de ses turbinespompes, GEC ALSTHOM Neyrpic a acquis une large expérience de ce type de machines. Les projets les plus récents auxquels la société a participé sont situés en Asie. Ce sont les projets de Guangzhou en Chine, Muju et San Chong en Corée. Les caractéristiques spécifiques de chacun de ces projets sont résumées dans le tableau 1 .

Tableau 1.

\begin{tabular}{|l|c|c|c|}
\hline \multicolumn{4}{|c|}{ PRINCIPALES CARACTÉRISTIQUES } \\
\hline & $\begin{array}{c}\text { Guang- } \\
\text { zhou }\end{array}$ & Muju & $\begin{array}{c}\text { San } \\
\text { Chong }\end{array}$ \\
\hline Nombres de groupes & 4 & 2 & 2 \\
Vitesse (tr/mn) & 500 & 450 & 360 \\
Chute nette turbine (m) & 537 & 580 & 423 \\
Puissance turbine (MW) & 348 & 336 & 391 \\
Chute nette pompe (m) & 537 & 601 & 431 \\
Puissance pompe (MW) & 326 & 330 & 364 \\
\hline
\end{tabular}

La centrale de Guangzhou réalisée pour Guangzhou Pumped Storage Power Station Joint Venture Corporation (GPSPSJV) a deux retenues de différents niveaux, avec une chute de $520 \mathrm{~m}$. Ces retenues sont reliées par un tunnel à une centrale électrique souterraine abritant quatre groupes hydroélectriques et quatre robinets sphériques. Des vannes wagons de secours sont installées en aval dans une caverne séparée. Chaque groupe est composé principalement d'une turbine-pompe réversible à un étage, et d'un alternateur moteur de 333 MVA (fig. I).

Cette centrale hydroélectrique est capable de convertir les $1300 \mathrm{MW}$ de la centrale électrique de Daya Bay, en puissance de pointe.

La centrale de Muju réalisée pour Korea Electricity Power Corporation (KEPCO) a deux retenues de différents niveaux, avec une chute de $589 \mathrm{~m}$. Ces retenues sont reliées par un tunnel à une centrale électrique souterraine abritant deux groupes hydroélectriques et deux robinets 


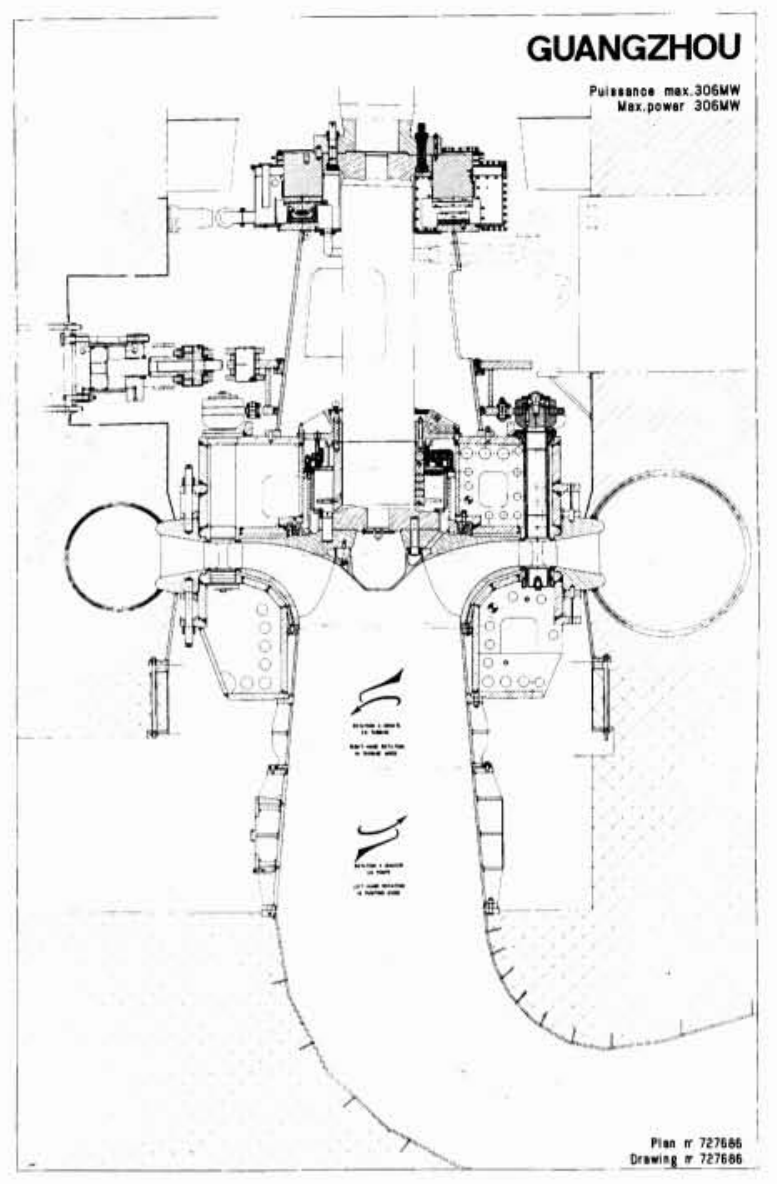

1. Coupe de la machine de Guangzhou.

sphériques. Chaque groupe est composé principalement d'une turbine-pompe réversible à un étage, et d'un alternateur moteur de 343 MVA (fig. 2).

La Centrale de Sanchong qui est en cours de réalisation pour Korea Electricity Power Corporation (KEPCO) a également deux retenues de différents niveaux, avec une chute de $427 \mathrm{~m}$. Ces retenues sont reliées par un tunnel à une centrale électrique souterraine abritant deux groupes hydroélectriques et deux robinets sphériques. Chaque groupe est composé principalement d'une turbine-pompe réversible à un étage, et d'un alternateur moteur de 405 MVA (fig. 3).

Tous les groupes de ces trois centrales sont capables de fonctionner en compensateurs synchrones et en turbine. Le démarrage en pompe se fait normalement par convertisseur statique de fréquence, avec possibilité complémentaire de démarrage « dos-à-dos » à partir de la vitesse zéro en cas de panne du convertisseur.

\section{D LIGNE D'ARBRE}

Chaque groupe a un arbre vertical et trois paliers, un sur le flasque supérieur de la turbine-pompe, les deux autres sont implantés de part et d'autre du rotor alternateur.

A Guangzhou, le palier turbine est un palier immergé à cuve tournante. Il est conçu pour présenter une très forte

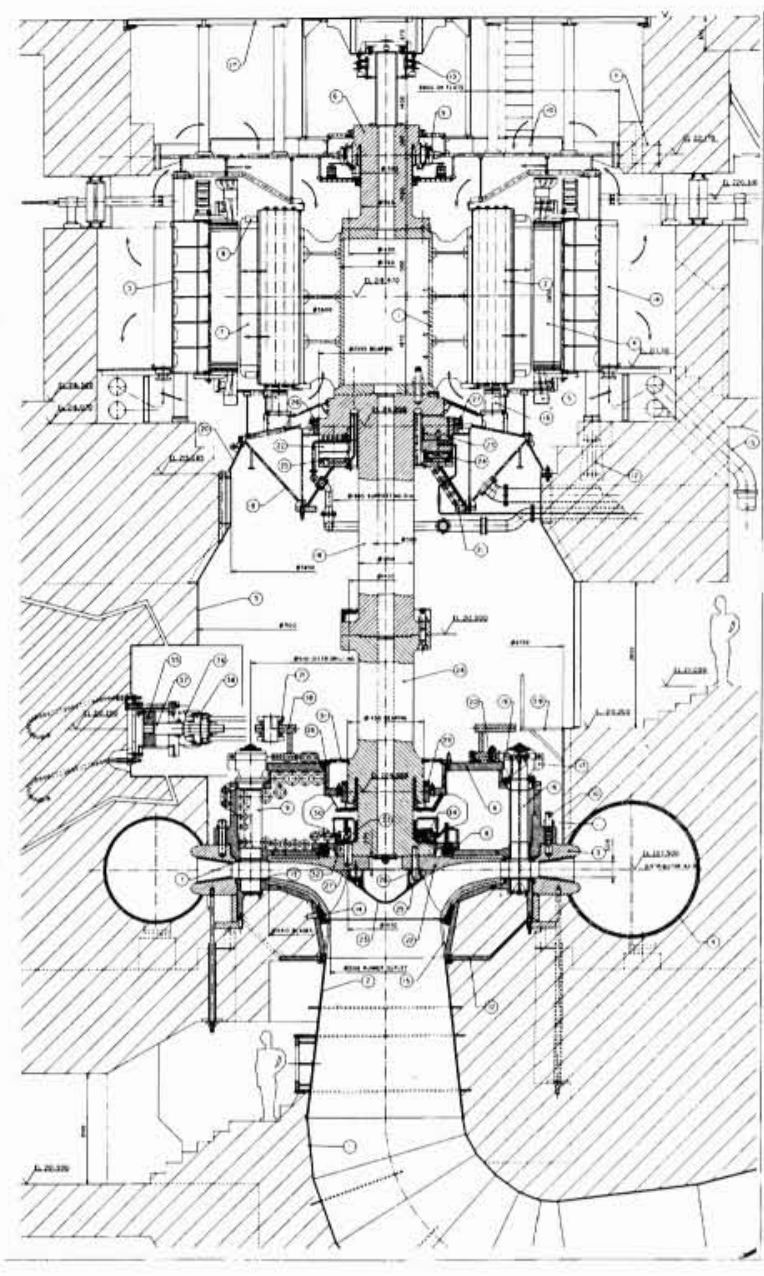

2. Coupe de la machine de Muju.

rigidité et absorber de très fortes charges dynamiques durant des transitoires. Il est fixé au flasque supérieur et ses réactions sont transmises aux ouvrages en béton par l'intermédiaire de l'avant-distributeur.

A Muju et San Chong, il s'agit de paliers classiques à patins avec dans les deux cas huit patins. Le joint d'arbre est situé au-dessous du palier sur le plateau de l'arbre.

\section{PIVOT}

A Guangzhou le pivot de technologie NEYRPIC est installé sur le flasque supérieur. De cette façon, on profite de la très grande rigidité de ce flasque, dimensionné pour résister aux charges hydrodynamiques de l'écoulement.

Il est calculé pour un effort axial d'un maximum transitoire de 980 tonnes. Il est composé d'un manchon monobloc qui repose sur 10 patins équilibrés par un circuit oléohydraulique, selon la technologie NEYRPIC à membranes autocompensées garantissant une équirépartition parfaite de la charge sur les patins. La circulation et le refroidissement de l'huile sont assurés par deux pompes et deux échangeurs situés à l'extérieur du puits turbine, dont un de secours. 


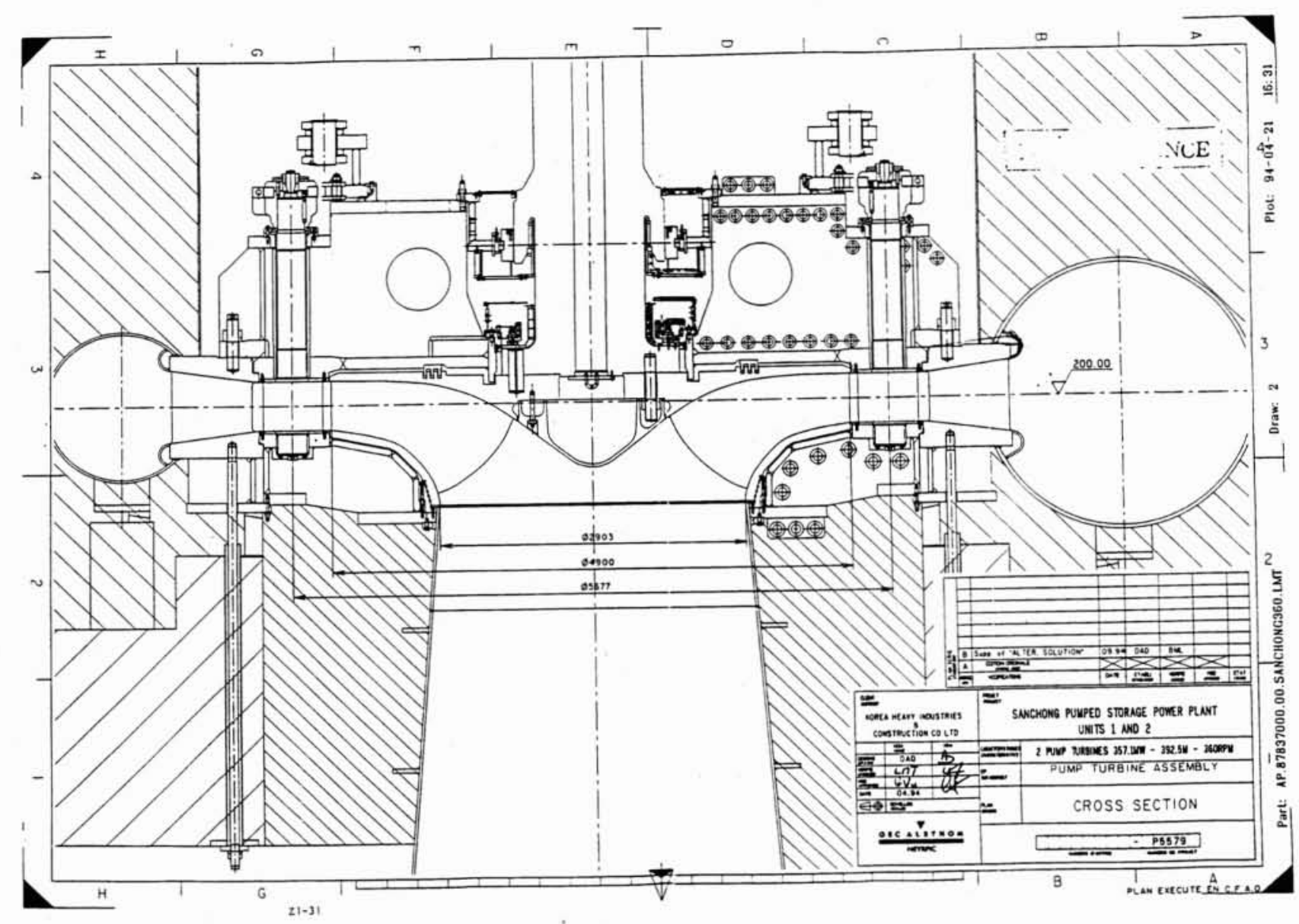

3. Centrale de pompage de San Chong.

A Muju et San Chong, le pivot est du type pivot-palier combiné en caisson. Il est installé sur le croisillon intermédiaire avec glace en deux parties qui repose comme à Guangzhou sur 10 patins équilibrés par un circuit oléohydraulique. Le pivot de Muju est calculé pour un effort axial maximum transitoire de 1325 tonnes et celui de Sanchong pour un effort de 1450 tonnes. Plus compacts que celui de Guangzhou, les pivots de Muju et San Chong dissipent moins d'énergie.

D'une manière générale la position du pivot est choisie en fonction des demandes du client.

\section{III $\square$ ROUE}

La roue qui compte 7 aubes à Guangzhou (fig. 4) et San Chong et 9 à Muju, est calculée par éléments finis. Elle est en acier inoxydable moulé. Elle est assemblée à l'arbre par goujons précontraints et pions de reprise de couple.

A Guangzhou, elle peut être démontée par dessous, ce qui réduit le temps de contrôle pendant l'entretien. Si nécessaire, la roue et les composants de l'anneau inférieur du distributeur, peuvent être sortis à travers une galerie sous la bâche spirale.

A Muju et San Chong le démontage s'effectue pardessus à travers l'alternateur. Toute la partie inférieure peut

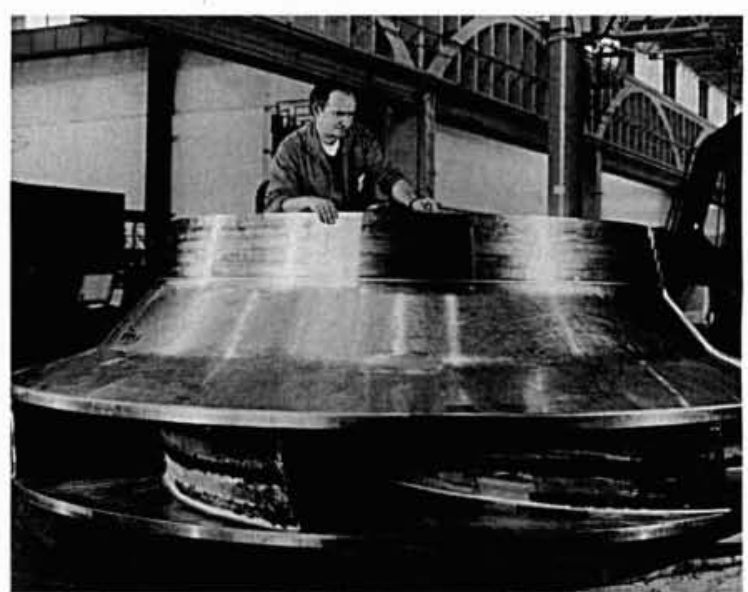

4. Roue de Guangzhou.

alors être bétonnée (aspirateur...), ce qui est plus favorable pour résister aux sollicitations dynamiques.

Les jeux aux labyrinthes étant réduits pour augmenter la performance des machines, les labyrinthes fixes sont exécutés en bronze pour éviter tout risque de grippage avec 
la roue lors du déclenchement d'un groupe, synonyme d'emballement et donc de déformation de la ligne d'arbre.

\section{DISTRIBUTEUR}

Le distributeur est un des sous-ensembles les plus sollicités de la machine. Il comprend principalement :

- deux flasques subissant chacun des efforts de pression de l'ordre de $15000 \mathrm{t}$ à Muju, $10000 \mathrm{t}$ à Guangzhou et de $7000 \mathrm{t}$ à San Chong.

- 20 directrices en acier inoxydable, mécaniquement synchronisées, commandées par un cercle de vannage actionné par deux servomoteurs. Chaque directrice est tenue par trois paliers équipés de bagues de bronze, avec graissage centralisé pour éviter tout risque de grippage par micro-déplacement. La durée de vie est meilleure avec des bagues en bronze qu' avec des matériaux auto-lubrifiants en particulier pour des turbines-pompes de haute chute.

Le fût des directrices est dimensionné pour éviter les fréquences d'excitation provoquées par le passage des aubes de la roue.

L'anneau inférieur qui doit être démonté à Guangzhou lorsqu'on veut démonter la turbine, est réalisé en quatre parties. A Muju et San Chong il est scellé.

L'ensemble est vérifié grâce à un programme de calcul développé par GANP, FLAXYPRO et également par la méthode des éléments finis en ce qui concerne les flasques.

Ce programme FLAXYPRO concerne la conception et le dimensionnement d'ensembles flasques, anneaux, directrices de turbines-pompes. Francis et Kaplan en mode graphique interactif. Trois étapes sont suivies :

- la conception géométrique des formes à partir de volumes élémentaires.

- une modélisation mécanique au cours de laquelle l'ensemble géométrique est transformé en un squelette fait d'unités mécaniques dont l'épaisseur est calculée automatiquement,

- après définition des charges mécaniques, le calcul est effectué à partir des équations de déformations au point de liaison des différentes structures.

Les charges dans les différentes parties des flasques et du distributeur ainsi que l'amplitude des déformations respectives sont précisément vérifiées.

\section{Vロ ENSEMBLE BÂCHE SPIRALE/AVANT- DISTRIBUTEUR}

L'ensemble bâche spirale/avant-distributeur doit remplir différentes fonctions. A côté de ses fonctions hydrauliques, il a un rôle important d'ancrage du groupe. Il doit assurer une très grande rigidité pour différentes raisons. Premièrement, du fait que les charges dynamiques transférées par la roue et le distributeur sont élevées durant les phases transitoires. Deuxièmement, la position du palier turbine doit être très précise pour éviter tout défaut d'alignement, les autres paliers étant fixés au béton du puits alternateur.

Les charges prises en compte pour le dimensionnement de l'ensemble sont la pression hydrostatique avec une pression d'essai avant bétonnage et les forces transmises par les flasques en fonctionnement.
Le calcul de l'épaisseur de la tôle de la volute nécessite une analyse poussée du champ de contraintes et en particulier durant les essais de pression. Un programme de calcul spécifique, développé par GANP, ProBac basé sur le comportement des coques toroïdales, est utilisé pour cette étude.

Cet ensemble est réalisé en morceaux à cause des limitations de transport. Les volutes sont soudées en atelier sur 2 demi avant-distributeurs et détensionnées. A Guangzhou (fig. 5) l'avant-distributeur est moulé en acier à haute limite élastique (400 MPa), à Muju et San Chong il est mécanosoudé ; il comprend deux anneaux en tôle et des avant-directrices en acier à haute limite élastique (450 MPa). Le travail sur le site consiste à raccorder par soudure les deux sections et à ajouter aux joints les deux viroles restantes également en acier à haute limite élastique (500 MPa à Guangzhou, $450 \mathrm{MPa}$ à Muju et $690 \mathrm{MPa}$ pour San Chong).

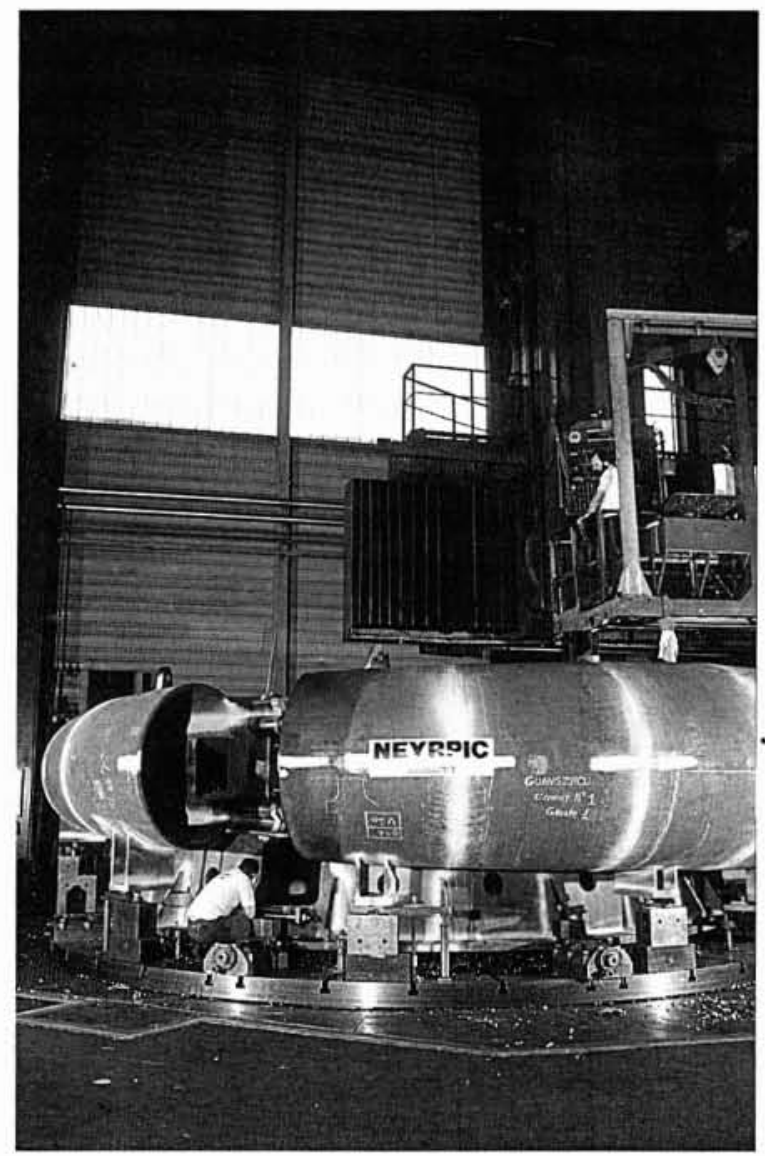

5. Bâche de Guangzhou.

A Guangzhou, l'ensemble pèse environ 170 tonnes et sa longueur hors-tout est de $10500 \mathrm{~mm}$ avec une bâche spirale d'un diamètre intérieur de $2300 \mathrm{~mm}$.

A Muju il pèse environ 230 tonnes et sa longueur horstout est de $13000 \mathrm{~mm}$ avec une bâche spirale d'un diamètre intérieur de $2250 \mathrm{~mm}$. 


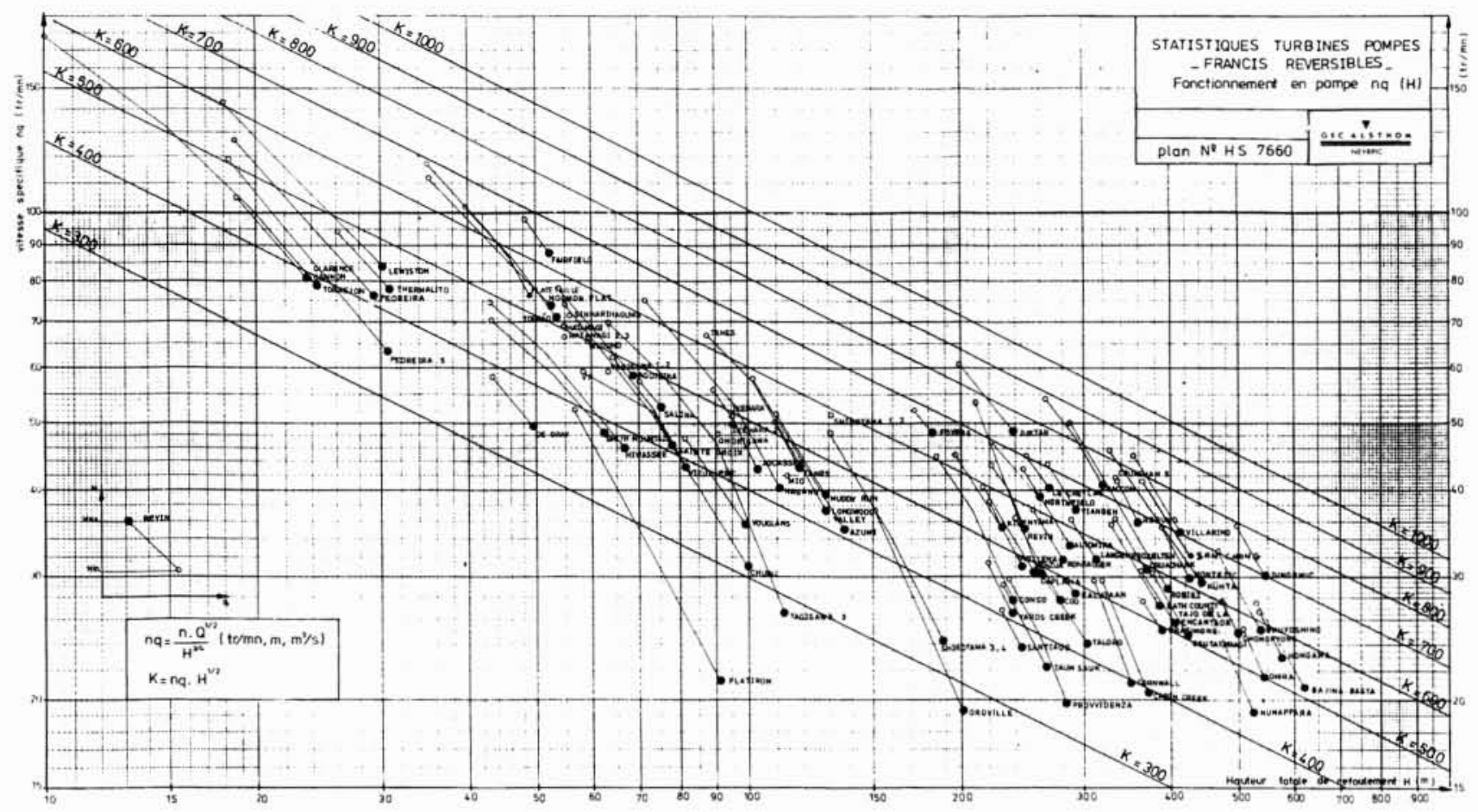

\section{Diagramme vitesse spécifique en pompe/hauteur de refoulement.}

A San Chong il pèse environ 240 tonnes et sa longueur hors-tout est de $13200 \mathrm{~mm}$ avec une bâche spirale d'un diamètre intérieur de $2875 \mathrm{~mm}$.

L'avant-distributeur comporte 20 avant-directrices qui relient deux anneaux d'une épaisseur d'environ $320 \mathrm{~mm}$. A Guangzhou, il possède à sa partie inférieure un cône soudé d'épaisseur $40 \mathrm{~mm}$ avec brides et nervures. Ce cône est fixé dans le béton avec 40 tiges précontraintes donnant un effort d'environ $100 \mathrm{t}$. Cette liaison est nécessaire pour transmettre au béton les efforts des groupes lors de certaines phases transitoires au cours desquelles la bâche est à pression réduite ( 8 à 12 bar). A Muju et San Chong, il est en appui sur le flasque inférieur par l'intermédiaire d'une structure mécanosoudée. Les efforts sont repris par des tirants d'ancrage précontraints. Les valeurs maximales transitoires sont de l'ordre de $4000 \mathrm{t}$ à Sanchong et de $11000 \mathrm{t}$ à Muju.

Il est possible d'accéder à l'intérieur du flasque supérieur de type en caisson par la virole extérieure à Guangzhou et par deux trous d'hommes situés sur la bride supérieure à Muju et San Chong. Cela permet de resserrer les brides de raccordement, d'ajuster les labyrinthes et de vérifier leurs jeux.

Le flasque inférieur est du type ouvert à Guangzhou, il offre un libre accès aux pots inférieurs des directrices. Ce fonds supporte les labyrinthes fixes inférieurs. A Muju et San Chong, le flasque inférieur est bétonné, en appui sur une structure mécanosoudée sous l'anneau de bâche inférieur. Les pots inférieurs des directrices ne sont pas accessibles.

Les fonds sont fixés aux avant-directrices par des goujons anti-soulèvement. A Guangzhou ces goujons sont pré-contraints par des vérins hydrauliques. A Muju et Sanchong cela n'est pas nécessaire car ces goujons subissent peu d'efforts du fait que le flasque inférieur est en appui sur l'anneau de bâche inférieur.

\section{D ROBINET SPHÉRIQUE}

A Guangzhou, le robinet sphérique est du type à obturateur prisonnier. Cette technologie qui permet un gain de poids est proposée par GEC ALSTHOM Neyrpic chaque fois que le robinet peut être transporté en une seule pièce. A Muju et Sanchong il est de conception classique avec à Sanchong un corps mécanosoudé.

Le robinet est équipé d'un joint en amont et aval. Un joint est manœuvré automatiquement pour chaque démarrage et arrêt. L'autre est manœuvré manuellement et peut être verrouillé mécaniquement pendant l'entretien. Le robinet sphérique est conçu pour tous les cas de fonctionnement y compris la fermeture du robinet sous un débit maximum.

\section{VII $\square$ CONCLUSION}

Le développement des turbines-pompes monoétages sous des chutes de plus en plus élevées s'est activement poursuivi au cours des 15 dernières années. Il s'est accompagné d'un développement technologique constant faisant appel à des capacités d'innovation et des compétences scientifiques et techniques de haut niveau. 
L'utilisation des turbines-pompes monoétages est envisageable jusqu'à des hauteurs de chutes de l'ordre de 700 à $800 \mathrm{~m}$. Actuellement les producteurs d'électricité commencent à évoquer l'utilisation de machines réglables sous des chutes supérieures à $800 \mathrm{~m}$. Pour de telles chutes, la turbine-pompe bi-étage à distributeur réglable semble la solution la plus intéressante.

En effet avec ce type de machines, la chute totale se répartit par moitié entre les 2 étages, ce qui présente plusieurs avantages :

- une vitesse spécifique plus élevée qui conduit à une hydraulique plus performante (meilleurs rendements, enfoncement plus faible) (fig. 6),

- la présence de l'arbre traversant l'aspirateur qui améliore considérablement le fonctionnement à charges partielles,

- des sollicitations mécaniques plus faibles. Les forces agissant sur la turbine-pompe (pulsation de pression et de couple sur les directrices, forces radiales sur les paliers) dépendant de la hauteur de refoulement induite par la roue, sont deux fois plus faibles,
- les aubes de roues sont plus accessibles pour le meulage.

Sur le plan économique cette solution est également intéressante car si les turbines-pompes bi-étages ont un coût de revient plus élevé que les turbines-pompes monoétages, elles conduisent malgré tout à un coût global d'installation inférieur. En effet leur mise en œuvre nécessite une caverne de plus faible dimension et un tunnel d'accès moins long grâce à un meilleur enfoncement des groupes.

Seule réalisation mondiale de ce type, le groupe expérimental de $37.7 \mathrm{MW}$ installé en France dans l'usine du Truel sous une chute de $450 \mathrm{~m}$ et mis en service en 1983 , permet à GEC ALSTHOM Neyrpic de disposer d'une expérience unique et approfondie autorisant une extrapolation sûre de ce type de machines vers des puissances décuplées et pour des chutes de 800 à $1000 \mathrm{~m}$. Fort de cette expérience et pour répondre aux attentes de ses clients, GEC ALSTHOM Neyrpic travaille actuellement sur des projets dans ces gammes de chute, pour de grandes puissances. 